previously been reported. We conclude that higher dosages of both preparations when administered together are effective and well tolerated in JHS patients with drug refractory POTS

\section{CATHETER ABLATION OF ATRIAL FIBRILLATION ON UNINTERRUPTED WARFARIN USING STANDARD AND DUTY CYCLED RADIOFREQUENCY ENERGY: SAFE AND EFFECTIVE}

doi:10.1136/heartjnl-2011-300198.161

J R J Foley, N C Davidson, B D Brown, D J Fox. University Hospital of South Manchester, Manchester, UK

Introduction Catheter ablation (CA) for atrial fibrillation (AF) is growing exponentially. Although ablation for paroxysmal AF (PAF) is associated with shorter procedure times and less extensive left atrial ablation vs persistent AF thromboembolic complications can occur in both sub-groups. Inadequate anticoagulation leads to thrombotic complications and excessive anticoagulation can lead to bleeding risks. Many centres adopt a policy of discontinuing warfarin in the immediate run-up to the procedure, covering the procedure with unfractionated heparin and "bridging" postoperative patients with low molecular weight heparins (LMWH) back onto warfarin. We wished to determine the safety of CA for AF with a therapeutic INR using both the single transseptal approach and duty cycled radiofrequency energy (RF) with non irrigated PVAC catheters and the double transseptal puncture technique using irrigated RF catheters and either CARTO or NAVX electroanatomical mapping.

Methods A retrospective analysis of 173 patients who underwent CA for AF while taking uninterrupted warfarin. Procedural target International Normalised Ratio (INR) was $2-3$ with a peri-procedural target ACT of $300-350 \mathrm{~s}$. In sub therapeutic INR patients weight adjusted LMWH was used post procedure with warfarin until INR was $>2$. Standard technique employed was large area circumferential ablation using conventional RF energy or pulmonary vein isolation using duty cycled RF energy. Data was gathered for demographics, procedural INR, total dose of unfractionated heparin, fluoroscopy time, and type of radiofrequency energy used. Endpoints were minor bleeding, major bleeding (requiring transfusion), vascular complications, pericardial tamponade and stroke/ TIA within 28 days of the procedure.

Results There were 128/173 male patients, age range between 21 and 73 years (mean 57 years). 122 underwent ablation for PAF and 51 for persistent AF. Mean procedural INR was 2.4 (range 1.7-3.9). Mean unfractionated heparin dose was 6000 units (range 1000-14500). Mean fluoroscopy time for the PVAC group was 23.4 mins (range 8.3-50.1 mins). Mean fluoroscopy time for CARTO/NAVX group was $31 \mathrm{mins}$ (range $14.10-58.44 \mathrm{mins}$ ). There were no major bleeding complications. There was 1 minor bleeding complication with a groin pseudoaneurysm. There were 2 cases of pericardial tamponade $(2 / 173 \%-1.2 \%)$ both managed with percutaneous pericardial drainage. There were no stroke/TIAs.

Conclusion These data demonstrate that CA for AF by both single and double transseptal technique using both standard RF and duty cycled RF while maintaining a therapeutic INR is a safe procedure. Maintaining a therapeutic INR reduces the risk of embolic events associated with "bridging" heparin without an increase in bleeding complications. This technique is convenient for patients and avoids switching between LMWH and warfarin and ensures patient safety by maintaining therapeutic anticoagulation before, during and after the procedure.

\section{2 THE CLINICAL MANAGEMENT OF RELATIVES OF YOUNG SUDDEN ARRHYTHMIC DEATH VICTIMS; ICDS ARE RARELY INDICATED}

doi:10.1136/heartjnl-2011-300198.162

${ }^{1} \mathrm{~J} \mathrm{C}$ Caldwell, ${ }^{1} \mathrm{Z}$ Borbas, ${ }^{2} \mathrm{~N}$ Moreton, ${ }^{2} \mathrm{~N}$ Khan, ${ }^{2} \mathrm{~L}$ Kerzin-Storrar, ${ }^{2} \mathrm{~K}$ Metcalfe, ${ }^{2} \mathrm{~W}$ Newman, ${ }^{1} \mathrm{C} \mathrm{J}$ Garratt. ${ }^{1}$ Manchester Heart Centre, Central Manchester University NHS Foundation Trust, Manchester, UK; ${ }^{2}$ Department of Clinical Genetics, St Mary's Hospital, Central Manchester University NHS Foundation Trust, Manchester, UK

Introduction Following National Service Framework guidance on the management of sudden cardiac death (SCD), regional inherited cardiac conditions clinics were established to identify and treat those at increased risk of dying from an arrhythmic condition. Studies have examined the yield of different diagnostic strategies but the outcome of management in these patients has not been reported.

Methods We present data on 193 consecutive patients (108 families) referred to a regional inherited cardiac conditions clinic because of $\mathrm{SCD} /$ aborted cardiac arrest of a relative. The mean age on referral was $38 \pm 17$ yrs and mean duration of follow-up was 15 months (range 1 day to 56 months). All individuals underwent clinical assessment by history, examination, ECG and echo. If treadmill exercise test had not previously been performed this was undertaken. Further imaging by CMR or contrast echo was performed in those with structurally abnormal hearts or with Twave inversion in $\mathrm{V}_{2} / \mathrm{V}_{3}$. Ajmaline provocative testing was performed in those with a history and/or ECG suggestive of Brugada syndrome.

Results Of the 193 patients, 43 individuals (22\%) from 36 families $(33 \%)$ were diagnosed with an inheritable cause of SCD and 145 patients were clinically normal (see Abstract 162 table 1). Five patients were found to have other conditions (LV non-compaction, AVNRT, skeletal myopathy, mild AS and congenital sub-aortic membrane). Of the 43 patients diagnosed with an inheritable condition, 21 had medication commenced by the clinic ( $\beta$-blockers (21), ACEi/ARB(2), Spironolactone[1]). ICDs were implanted as per HRUK guidelines, resulting in 4 patients having an ICD inserted on clinic recommendation (2 HCM, 1 DCM, 1 ARVC). To date no appropriate therapies have been administered (follow-up 8-29 months) but there was 1 inappropriate shock from a fractured lead. Three individuals had $\beta$-blockade withdrawn after negative genetic testing for an established familial mutations (2 CPVT, 1 LOT), one ICD was removed and one deactivated (both negative for CPVT). Of the 145 patients thought to be clinically normal, 85 were reassured and discharged, 13 failed to return to clinic and 47 are regular reviewed as the risk of developing an inheritable condition cannot be excluded; this includes those with family histories of HCM (7), ARVC (12), DCM (9), CPVT (5), Brugada (4) and LOT(1). To date no deaths have occurred in those diagnosed with inherited causes of SCD (follow-up mean 20, 1-52 range) or those clinically normal with ongoing review (follow-up mean 22 months, 1-56 range).

\section{Abstract 162 Table 1}

\begin{tabular}{lc}
\hline Diagnosis of patient & Numbers \\
\hline Clinically normal & 145 \\
LOTS & 12 \\
Brugada & 2 \\
CPVT & 5 \\
ARVC & 7 \\
DCM & 7 \\
HCM & 10 \\
\hline
\end{tabular}

Conclusion A diagnosis of an inheritable cause of SCD was obtained in $22 \%$ of individuals and $33 \%$ families with a history of SCD/ aborted cardiac arrest in a relative. The number of ICDs inserted was very small $(2 \%)$ and there have been no appropriate therapies in this 
group. Two ICDs have been removed/deactivated after exclusion of a known familial mutation.

\section{THE UNITED KINGDOM TRANSCATHETER AORTIC VALVE REGISTRY - OUTCOMES TO DECEMBER 2009 AND UPDATE}

doi:10.1136/heartjnl-2011-300198.163

P F Ludman. On Behalf of the UK TAVI Steering Group, Birmingham, UK

Introduction The United Kingdom Transcatheter Aortic Valve (TAVI) Registry was established to define the clinical and procedural details of all patients being treated by TAVI, regardless of access route or technology used, and to assess their outcomes. The registry has captured all cases in England and Wales.

Methods For every TAVI performed, all centres complete an agreed dataset. The data are encrypted and sent electronically to servers at the Central Cardiac Audit Database (CCAD) for analysis. A unique patient identifier (the NHS number) is used to link with the NHS Central Register to track mortality. This analysis is based on all procedures performed up to 31st December 2009

Results 25 centres in England and Wales performed a total of 877 procedures in 870 patients; 66 in 2007, 273 in 2008 and 538 in 2009. Median number of cases per centre was 24. Median (IOR) age was 82 (78-87) yrs. 52\% were male, and mean logistic Euroscore (LES) was $22.2 \%$. $68 \%$ were transfemoral, the remainder being mainly transapical. $90 \%$ of CoreValve implants and $46 \%$ of Edwards used a transfemoral approach. Patients needing a transapical route had more comorbid conditions than those treated by a transfemoral route (LES $25.2 \%$ vs $20.9 \%$ ). Mortality tracking was successful in $100 \%$ of patients. Survival at 30 days was $93.1 \%, 78.9 \%$ at 1 year (443 at risk) and $72.3 \%$ at 2 years (114 at risk). Survival was significantly poorer in those needing non-transfemoral approaches (1 year survival $73.5 \%$ vs $81.4 \%$ ). Survival was also poorer in those with poorer LV ejection fraction, with moderate or severe post procedural aortic regurgitation and with a LES $>40$. Survival was not associated with age, NYHA class, or the presence of concomitant coronary artery disease, and not different between those with a LES $<21$ compared with LES 21-40. There was also no difference in survival between patients treated with CoreValve or Edwards technologies, or between proctored and non proctored cases. There was a significant improvement in survival over the 3 years of the registry, and a change in demographics with the proportion of patients with prior CABG rising from $16.4 \%$ in 2007 to $29.9 \%$ in 2009. The total number of cases in the UK TAVI registry has risen to 1490 as of 29 Nov 2010. More details of the 2010 cohort will be available at the time of presentation (Abstract 163 figure 1).

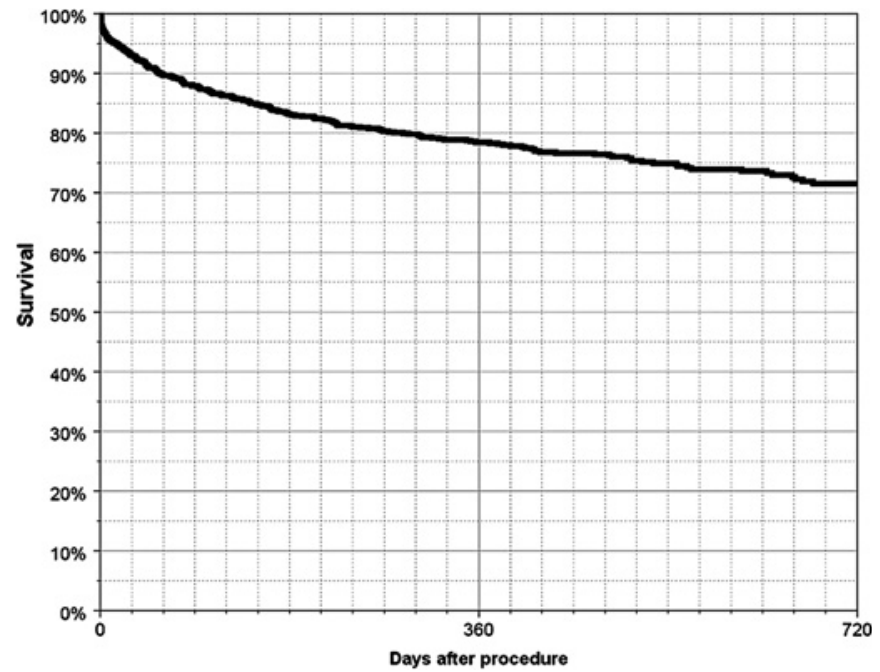

Abstract 163 Figure 1
Conclusions The UK TAVI Registry has successfully captured the entire TAVI activity of England and Wales incorporating the learning curves of every centre. Outcomes following TAVI are excellent in a high risk patient population. Outcomes are better in the population who are suitable for a transfemoral approach (with less comorbidity) than those treated with the transapical approach. These data suggest that careful proctoring allows the introduction of a new treatment method without an adverse effect on patient outcome, and we have demonstrated no systematic difference in outcome between the two commercially available technologies.

\section{EARLY HAEMODYNAMIC CHANGES AND MYOCARDIAL INJURY AFTER TRANSFEMORAL TRANSCATHETER AORTIC VALVE IMPLANTATION (TAVI)}

doi:10.1136/heartjnl-2011-300198.164

R Dworakowski, A Bhan, B Brickham, O Wendler, M Monaghan, A M Shah, P MacCarthy. Kings College Hospital, Kings Health Partners, London, UK

Purpose Transfemoral (TF) TAVI is a novel procedure for the treatment of severe aortic stenosis, without the need for thoracotomy or cardiopulmonary bypass. The procedure results in almost instantaneous normalisation of transvalvular gradients, but little is yet known about the periprocedural haemodynamic effects. We aimed to describe these effects using 3D and tissue Doppler (tD) transthoracic echocardiography (TTE) and Cardiac Output monitoring. Methods In 16 patients undergoing TF TAVI haemodynamics were characterised with a number of $\mathrm{tD}$ and $3 \mathrm{D}$ TTE measurements.

A

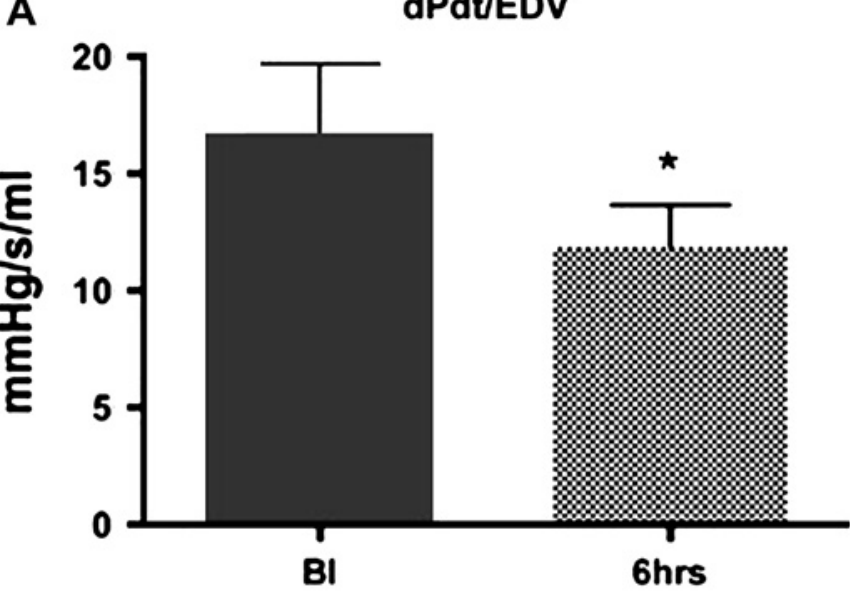

B E/E' medial

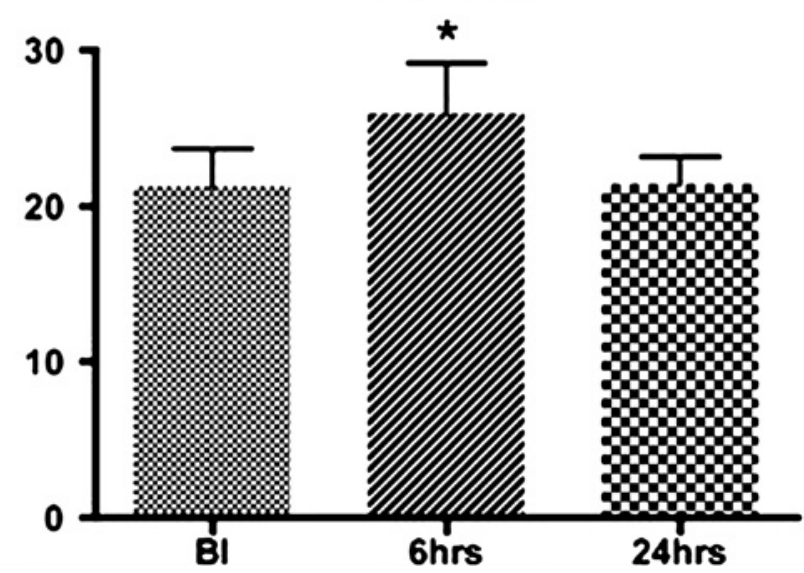

Abstract 164 Figure 1 